\title{
Constitutive expression of the wheat TaSOD5 gene enhances salinity tolerance of Arabidopsis thaliana
}

\author{
Y.-G. SONG, T.-X. GAO, X.-J. LIU, and W. DONG* \\ School of Life Science, Qufu Normal University, Qufu, Shandong, 273165, P.R. China
}

\begin{abstract}
Superoxide dismutase is a crucial reactive oxygen species (ROS) scavenger and converts the superoxide radical $\left(\mathrm{O}_{2}^{-}\right)$to $\mathrm{H}_{2} \mathrm{O}_{2}$, so it is thought to enhance abiotic stress tolerance by reducing ROS accumulation and so avoiding oxidative damage. In this study, we isolated a salt- and oxidative stress-responsive $\mathrm{Cu} / \mathrm{Zn}$ SOD gene TaSOD5 from wheat. The ectopic overexpression of TaSOD5 in Arabidopsis increased total and $\mathrm{Cu} / \mathrm{Zn}$ SOD activities, and offered the plant tolerance to salt stress. Arabidopsis ectopically expressing TaSOD5 possessed a superior resistance to oxidative stress induced $\mathrm{H}_{2} \mathrm{O}_{2}$. The TaSOD5 ectopic overexpression elevated the activities of both ROS scavengers and $\mathrm{O}_{2}{ }^{-}$producer NADPH oxidase. These findings show that $\mathrm{Cu} / \mathrm{Zn}$ SOD enhances salt tolerance via regulating the machinery of redox homeostasis rather than improving SOD activity alone.
\end{abstract}

Additional key words: APX, CAT, GPX, NADPH oxidase, redox homeostasis, ROS, superoxide dismutase, Triticum aestivum.

\section{Introduction}

Soil salinity has a significant limit on crop productivity; it has been estimated that nearly a half of the irrigated areas and about $20 \%$ of the arable land in the world are affected by a varying degree of salinity (Dong et al. 2018). Salt stress produces some primary damage to ion balance, water relations, photosynthetic efficiency, and nutrient absorption, and then extends to a secondary damage due to ion toxicity and overproduction of reactive oxygen species (ROS) (Ihsan et al. 2016). One source of ROS production is the reduction of $\mathrm{O}_{2}$, which is excitated to form singlet oxygen $\left(\mathrm{O}_{2}{ }^{1}\right)$ or is transferred with electron(s) to form the superoxide radical $\left(\mathrm{O}_{2}^{-}\right)$(Mittler 2002). To cope with the damage by ROS, plants have developed efficient ROS scavenging machinery consisting of a set of nonenzymatic compounds and enzymes including superoxide dismutase (SOD), catalase (CAT), and ascorbate peroxidase (APX) (Mittler 2002). Superoxide dismutase serves as the first defense line to convert $\mathrm{O}_{2}^{-}$to hydrogen peroxide $\left(\mathrm{H}_{2} \mathrm{O}_{2}\right)$ (Fridovich 1995). The SOD family comprises three classes, $\mathrm{Cu} / \mathrm{Zn}$-SOD), Fe-SOD, and Mn-SOD. Of them, $\mathrm{Cu} / \mathrm{Zn}-\mathrm{SOD}$ has been found to enhance tolerance to abiotic stresses in plants (Allen 1995, Gill et al. 2015).
On the other hand, a suitable ROS amount is necessary for triggering response to environmental stimuli, and ROS play crucial regulatory performance in numerous biological processes (Mittler et al. 2011, Suzuki et al. 2012, Sierla et al. 2013). Reactive oxygen species amount is governed by homeostasis between ROS production and scavenging pathways (Miller et al. 2009). However, only the improvement of ROS scavenging could not ensure the enhancement of tolerance to abiotic stresses (Pitcher et al. 1991). These findings suggest the roles of SOD and other ROS scavengers in salt tolerance are performed via mechanisms more complicated than we expect, which has not been well addressed so far.

Common wheat (Triticum aestivum L.) is one of the most important food crops, but it has only limited resistance to salinity stress (Kumar et al. 2018). Salinity hinders leaf growth and tillering by halting the leaf primordia initiation rates and finally lead to reduction of various yield parameters including kernel mass and number (Francois et al. 1994). In the past decades, important advances have been made in the understanding of the mechanism of salinity tolerance in wheat (Kumar et al. 2018). Wheat plants mitigate the effects of salinity stress by up-regulating various stress-responsive genes,

Submitted 19 March 2019, last revision 27 July 2019, accepted 21 August 2019.

Abbreviations: APX - ascorbate peroxidase; CAT - catalase; GPX - glutathione peroxidase; NOX - NADPH oxidase; PEG - polyethylene glycol; OE - transgenic Arabidopsis lines over-expressing a superoxide dismutase gene TaSOD5; ROS - reactive oxygen species; SOD - superoxide dismutase.

Acknowledgements: This research was financially supported by the National Natural Science Foundation of China (grant Nos 31501328 and 31300220) and the Natural Science Foundation of Shandong (ZR2015JL012 and ZR2014CP013).

* Corresponding author; e-mail: dwei@qfnu.edu.cn 
including ion transporters, transcription factors, signaling pathway regulators, and antioxidant enzymes. (Dong et al. 2013, Ge et al. 2013, Liu et al. 2014, Rong et al. 2014, Huang et al. 2015, Zahid et al. 2017). Dynamic expression of these genes authenticates their inevitability and sufficiency in enhancing salt tolerance in wheat. For example, TaHKT1 plays a key role in $\mathrm{Na}^{+} / \mathrm{K}^{+}$balance at a high concentration of $\mathrm{Na}^{+}$, and Triticum aestivum Superoxide dismutase $1 / 2$ can crosstalk with other proteins to participate in various signaling pathways (Kumar et al. 2018). However, there are few reports on the regulation of salt stress by balancing ROS production and scavenging systems in wheat.

The aim of this research was to identify a stress responsive $\mathrm{Cu} / \mathrm{Zn}$ SOD gene TaSOD5 from the salt tolerant wheat and determine if TaSOD5 ectopic overexpression in Arabidopsis can improve the activities of some ROS scavengers and enhance the salinity tolerance via altering ROS homeostasis. Discovery and functional identification of this gene might be a potential target for determination of salinity-tolerant and susceptible cultivars for molecular breeding studies in the future.

\section{Materials and methods}

Plants and treatments: Two common wheat (Triticum aestivum.L.) cultivars, Shi8 with a remarkable salinity tolerance, and Yimai38 which shows salt intolerance were grown in a half strength Hoagland liquid medium (Hoagland and Arnon 1950) in a ceramic pot, with small holes in the lid, under a $16-\mathrm{h}$ photoperiod, an irradiance of $300 \mu \mathrm{mol} \mathrm{m} \mathrm{m}^{-2} \mathrm{~s}^{-1}$, a temperature of $22{ }^{\circ} \mathrm{C}$, and an air humidity of $55 \%$. For stress treatments, two-week-old seedlings were transferred to media containing $200 \mathrm{mM}$ $\mathrm{NaCl}, 18 \mathrm{~g} \mathrm{dm}^{-3}$ polyethylene glycol (PEG) 6000, $10 \mathrm{mM}$ $\mathrm{H}_{2} \mathrm{O}_{2}$, or $100 \mu \mathrm{M}$ abscisic acid for $0,1,3,6,12$, and $24 \mathrm{~h}$.

Arabidopsis thaliana L. seeds harvested from TaSOD5 transgene homozygous plants (OE1 and OE2 lines) and wild type(Col-0)were surface-sterilized and plated on a solidified Murashige and Skoog (1962 agar medium and kept in the dark at $4{ }^{\circ} \mathrm{C}$ for $3 \mathrm{~d}$ to break dormancy, and then transferred to a 16-h photoperiod, an irradiance of $300 \mu \mathrm{mol} \mathrm{m} \mathrm{m}^{-2} \mathrm{~s}^{-1}$, and a temperature of $22{ }^{\circ} \mathrm{C}$ for $2 \mathrm{~d}$. Then, they were replated on half strength Murashige and Skoog agar media supplemented with various concentrations of $\mathrm{NaCl}$ for $14 \mathrm{~d}$, or $\mathrm{H}_{2} \mathrm{O}_{2}$ for $10 \mathrm{~d}$. Each treatment was replicated three times. Plant tissues for RNA extraction were snap-frozen in liquid nitrogen and stored at $-80^{\circ} \mathrm{C}$ until required.

Isolation of TaSOD5: The sequence with annotation as $S O D$ for the RNA sequencing dataset was subject to BLASTN against the wheat EST database in NCBI. The matched sequences were assembled using the CAP3 software (Huang and Madan 1999). According to the assembled sequence, the full-length cDNA (named TaSOD5) from the common wheat drought tolerant cultivar Shi8 was isolated. The PCR procedure included a denaturation at $95^{\circ} \mathrm{C}$ for $5 \mathrm{~min}, 35$ cycles of $94^{\circ} \mathrm{C} / 30 \mathrm{~s}, 58^{\circ} \mathrm{C} / 50 \mathrm{~s}$, and $72^{\circ} \mathrm{C} / 60 \mathrm{~s}$, and a final extension at $72{ }^{\circ} \mathrm{C}$ for $10 \mathrm{~min}$. The amplified product was inserted into the pMD18-T vector (Takara, Dalian, China), and submitted for Sanger sequencing.

Construction of TaSOD5 transgenic Arabidopsis plants: The TaSOD5 was ligated into the pSTART vector to construct a pSTART-TaSOD5 vector driven by the CaMV:35S promoter. The pSTART-TaSOD5 vector was introduced into Arabidopsis Col-0 using the floral dip method (Clough and Bent 1998). Homozygous transgenic lines were selected by kanamycin screening. The DNA of the transgenic lines was extracted for PCR to detect the integration of TaSOD5. The RNA of the transgenic lines was isolated and reversely transcribed to produce cDNA, and cDNA was used as a template for real-time quantitative PCR to measure the expression of TaSOD5.

Total RNA was extracted from samples mentioned above using a Trizol reagent (Takara), and treated with DNAase I. The RNA was reversely transcribed to synthesize the first cDNA strand using an $M-M L V$ reverse transcription system kit (Takara). The cDNA was used as a template for realtime quantitative PCR in $0.02 \mathrm{~cm}^{3}$ of solution containing $10 \mathrm{~mm}^{3}$ of $2 \times$ SYBR Premix Ex Taq mix (Takara), $0.2 \mu \mathrm{M}$ forward (5'-GAGGTGAGGGGGAGGGTCTC-3') and $0.2 \mu \mathrm{M}$ reverse (5'-TCGTTCATCATCCATCGGTG-3') primers, $1 \mathrm{~mm}^{3}$ of a 1:10 diluted cDNA first strand, and the cycling regime comprised a denaturation step of $95{ }^{\circ} \mathrm{C} / 2 \mathrm{~min}$ followed by 45 cycles of $95{ }^{\circ} \mathrm{C} / 10 \mathrm{~s}$, $60{ }^{\circ} \mathrm{C} / 20 \mathrm{~s}$, and $72{ }^{\circ} \mathrm{C} / 20$ s. Melting curve analysis was performed over the range of 80 to $95{ }^{\circ} \mathrm{C}$ at $0.5^{\circ} \mathrm{C}$ intervals. Relative gene expressions were detected using the $2^{-\Delta \Delta C \mathrm{~T}}$ method (Livak and Schmittgen 2001). The wheat ACTIN gene (AB181991) or Arabidopsis TUBULIN gene (AT1G04820) were used as internal references.

Measurement of activities of ROS scavenging enzymes and $\mathrm{Na}^{+} / \mathbf{K}^{+}$content: The leaves of three-week-old wheat seedlings and two-week-old Arabidopsis seedlings were collected. The leaves $(0.5 \mathrm{~g})$ were homogenized in $1 \mathrm{~cm}^{3}$ of homogenization solution on ice. The solution consisted of $50 \mathrm{mM} \mathrm{KH}_{2} \mathrm{PO}_{4}, 0.1 \mathrm{mM} \mathrm{Na} \mathrm{FDTA}_{2}$, and $0.3 \%(\mathrm{v} / \mathrm{v})$ Triton $X-100$. The homogenate was centrifuged at $13000 \mathrm{~g}$ for $10 \mathrm{~min}$, and a certain volume of the protein extraction solution was sampled to measure enzyme activities. Protein content was determined using the Bradford Coomassie brilliant blue staining assay (Bradford 1976).

Total SOD activity was measured using the nitroblue tetrazolium method with a SOD activity kit; $\mathrm{Cu} / \mathrm{Zn}$ SOD activities was measured with a $\mathrm{Cu} /$ $\mathrm{Zn}-\mathrm{SOD}$ and Mn-SOD assay kit (Beyotime, Shanghai, China). Ascorbate peroxidase activity was measured according to the respective kit instructions (Jianchen, Nanjing, China). Catalase activity was measured by detecting a decrease in absorbance at $240 \mathrm{~nm}$ for 1 min using a CAT activity measurement kit (Beyotime). Glutathione peroxidase (GPX) activity was determined by monitoring a decrease in absorbance at $340 \mathrm{~nm}$ of a reaction system using a GPX activity measurement kit (Beyotime), and a coefficient of absorbance for NADPH 
was assumed as $6.22 \cdot 10^{3} \mathrm{M}^{-1} \mathrm{~cm}^{-1}$.

Content of $\mathrm{Na}^{+}$and $\mathrm{K}^{+}$was determined by an inductively coupled plasma atomic emission spectrometer (ICP-AES, Perkin-Elmer, Boston, MA, USA) according to Dong et al (2012).

Measurement of NADPH oxidase activity: Leaves $(0.5 \mathrm{~g})$ were sampled from two-week-old Arabidopsis seedlings, and homogenized in $1 \mathrm{~cm}^{3}$ of homogenization solution containing $50 \mathrm{mM} \mathrm{KH}_{2} \mathrm{PO}_{4}$ and $0.1 \mathrm{mM} \mathrm{Na} \mathrm{NDDT}_{2}$. The homogenate was centrifugated at $13000 \mathrm{~g}$ and $4{ }^{\circ} \mathrm{C}$ for $15 \mathrm{~min}$, and the supernatant was collected for measuring the activity of NADPH oxidase (NOX) according to Grace and Logan (1996). A reaction solution contained $100 \mathrm{mM}$ Tris-HCl, pH 8.0, 1 mM Na $\mathrm{EDTA}_{2}$, and $0.2 \mathrm{mM}$ NADPH. The reaction began when NADPH was added. Activity of NOX was calculated by monitoring a decrease in absorbance at $340 \mathrm{~nm}$. The molar coefficient of absorbance for NADPH was $6.22 \cdot 10^{3} \mathrm{M}^{-1} \mathrm{~cm}^{-1}$.

Statistics: Values are presented as means of three replicates and standard errors (SDs). Experimental data were analyzed by the SPSS software (IBM) followed by one-way $A N O V A$ to determine significant differences among treatments.

\section{Results}

The TaSOD5 encodes a protein possessing a $\mathrm{Cu} / \mathrm{Zn}$ SOD catalytic site and shares high identities with $\mathrm{Cu} /$ Zn SOD members from other species. To know whether TaSOD5 is involved in a response to abiotic stresses, the transcriptional profiles of TaSOD5 under several treatments were detected. After exposure to $200 \mathrm{mM} \mathrm{NaCl}$, TaSOD5 was gradually induced with a stronger induction in 'Shi8' than in its parent cultivar 'Yimai38' (Fig. 1). When treated with $18 \mathrm{~g} \mathrm{dm}^{-3}$ PEG 6000 solution to mimic osmotic stress, the expression of TaSOD5 was also elevated, and peaked at $12 \mathrm{~h}$; during the whole course of treatment, 'Shi8' accumulated more TaSOD5 transcripts than 'Yimai38' did (Fig. 1). When suffered from oxidative stress stimulated by applying exogenous $\mathrm{H}_{2} \mathrm{O}_{2}$, the transcription of TaSOD5 was only slightly higher in 'Shi8' than in 'Yimai38' (Fig. 1). The application of abscisic acid, an abiotic stress associated phytohormone, accelerated the expression of

\section{TaSOD5 especially in 'Shi8' (Fig. 1).}

To determine the role of TaSOD5 in the adaption to abiotic stresses, we constructed two TaSOD5 ectopic overexpression lines (OE1 and OE2) of Arabidopsis ecotype Col-0. The TaSOD5 was integrated in the genomes of the two transgenic lines, and their transcripts were detected (Fig. 2A,B). In comparison with Col-0, two OE lines had higher total SOD activities (Fig. 2C). Consistently, the $\mathrm{Cu} / \mathrm{Zn}$ SOD activities of the two OE lines were significantly elevated when compared with Col-0 (Fig. 2C). The results show that TaSOD5 encoded a $\mathrm{Cu} /$ $\mathrm{Zn} \mathrm{SOD,} \mathrm{and} \mathrm{its} \mathrm{ectopic} \mathrm{overexpression} \mathrm{improved} \mathrm{SOD}$ activity in Arabidopsis.

The two OE lines had no phenotypic alteration from Col-0 during the whole life course ranging from vegetative to reproductive growth in soil (data not shown) indicating that TaSOD5 had no effect on development of Arabidopsis plants. Similarly, the two OE lines had a comparable growth ability and phenotype to Col- 0 when growing on the agar plate (Fig. $3 A, B$ ). On the agar plate containing various concentration of $\mathrm{NaCl}$, the growth of seedlings was restricted, but the restriction was attenuated in the OE lines, which had longer roots and higher shoot masses than Col-0 (Fig. 3A,B). Salt stress can affect the ionic homeostasis between $\mathrm{Na}^{+}$and $\mathrm{K}^{+}$, and therefore destroy the physiological processes. We found that the content of $\mathrm{Na}^{+}$and the content of $\mathrm{K}^{+}$were both comparable between Col- 0 and the OE lines under the control conditions, and the ratios of $\mathrm{K}^{+}$to $\mathrm{Na}^{+}$were also similar among all plants (Fig. $3 B$ ). When subjected to $100 \mathrm{mM} \mathrm{NaCl}$, the content of $\mathrm{Na}^{+}$increased (Fig. 3B). In comparison with Col-0, the alterations in $\mathrm{Na}^{+}$and $\mathrm{K}^{+}$content were smaller in the $\mathrm{OE}$ lines, so that the $\mathrm{OE}$ lines had a lower $\mathrm{Na}^{+}$but higher $\mathrm{K}^{+}$content than Col- 0 did. These data indicate that TaSOD5 ectopic overexpression enhanced salt tolerance and ionic homeostasis maintenance ability in Arabidopsis.

Given that salt and other abiotic stresses often induce over-production of ROS, we further analyzed the role of TaSOD5 in the response to oxidative stress. After exposure to oxidative stress (by applying $\mathrm{H}_{2} \mathrm{O}_{2}$ ), seedling growth was restricted; the restriction was attenuated in the $\mathrm{OE}$ lines, which had longer roots and larger shoots than Col-0 (Fig. 4A,B). The evidence confirms that TaSOD5 ectopic overexpression enhanced tolerance to oxidative stress.

Superoxide dismutase is the central effector of the ROS scavenging machinery, in which the close link among

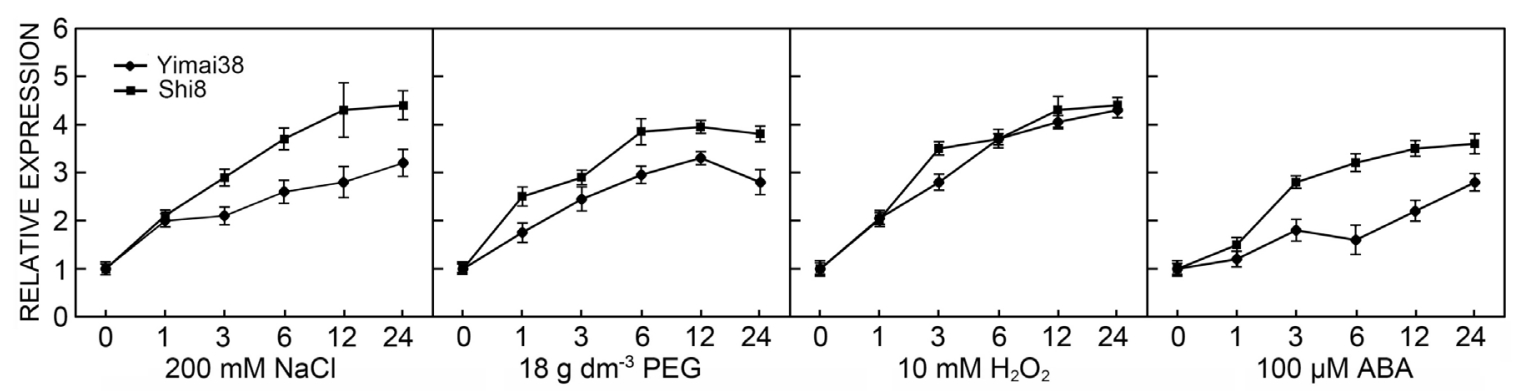

Fig. 1. Expression of a suporoxide dismutase gene TaSOD5 was responsive to abiotic stresses. Wheat seedlings at three-leaf-stage were subjected to $200 \mathrm{mM} \mathrm{NaCl}, 18 \mathrm{~g} \mathrm{dm}^{-3}$ polyethylene glycol (PEG) $6000,10 \mathrm{mM} \mathrm{H}_{2} \mathrm{O}_{2}$, or $100 \mathrm{M}$ abscisic acid (ABA) for 0 to $24 \mathrm{~h}$, and TaSOD 5 expressions were detected using real-time quantitative PCR. Means \pm SDs, $n=3$. 
components is present. Thus, we detected the activities of GPX, APX, and CAT and in comparison with Col-0, the OE lines had higher APX and GPX activities (Fig. 4C). Similarly, the OE lines had also a slightly higher CAT activity than Col-0 although the difference between Col-0 and OE2 was not significant $(P=0.0542)$ (Fig. $4 C)$. These data show that TaSOD5 ectopic overexpression enhanced the activity of ROS scavenging system in addition to SOD activity.

Suitable intracellular ROS production is essential for

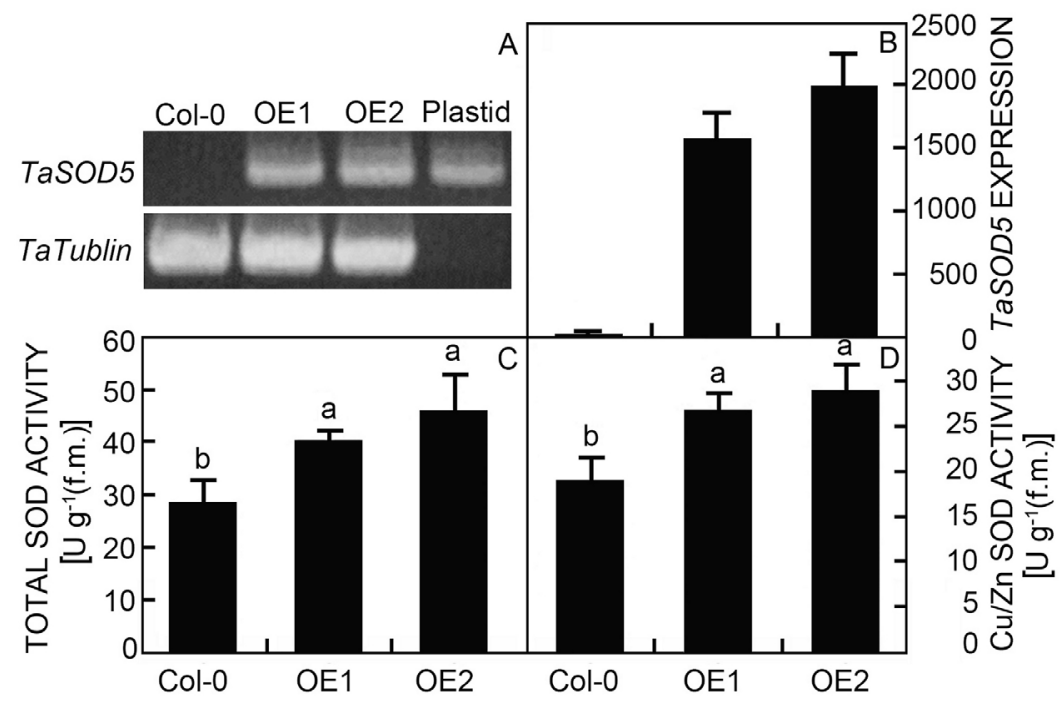

Fig. 2. Superoxide dismutase TaSOD5 ectopic overexpression improved SOD activity in Arabidopsis. A - PCR analysis indicates that TaSOD5 was integrated in the genome of Arabidopsis. B - Real-time PCR analysis indicates production of transcripts of TaSOD5 in Arabidopsis. $C$ - The total SOD and Cu/Zn SOD activities in Arabidopsis ecotype Col-0 and the transgenic Arabidopsis lines OE1 and OE2 ectopically expressing TaSOD5. (Plastid - the pSTART plastid fused with TaSOD5; U - the unit of SOD activity which is defined as enzyme activity when the inhibition of the xanthine oxidase is $50 \%$ ). Means \pm SDs, $n=3$, different letters indicate significant differences using one-way ANOVA $(P<0.05)$.
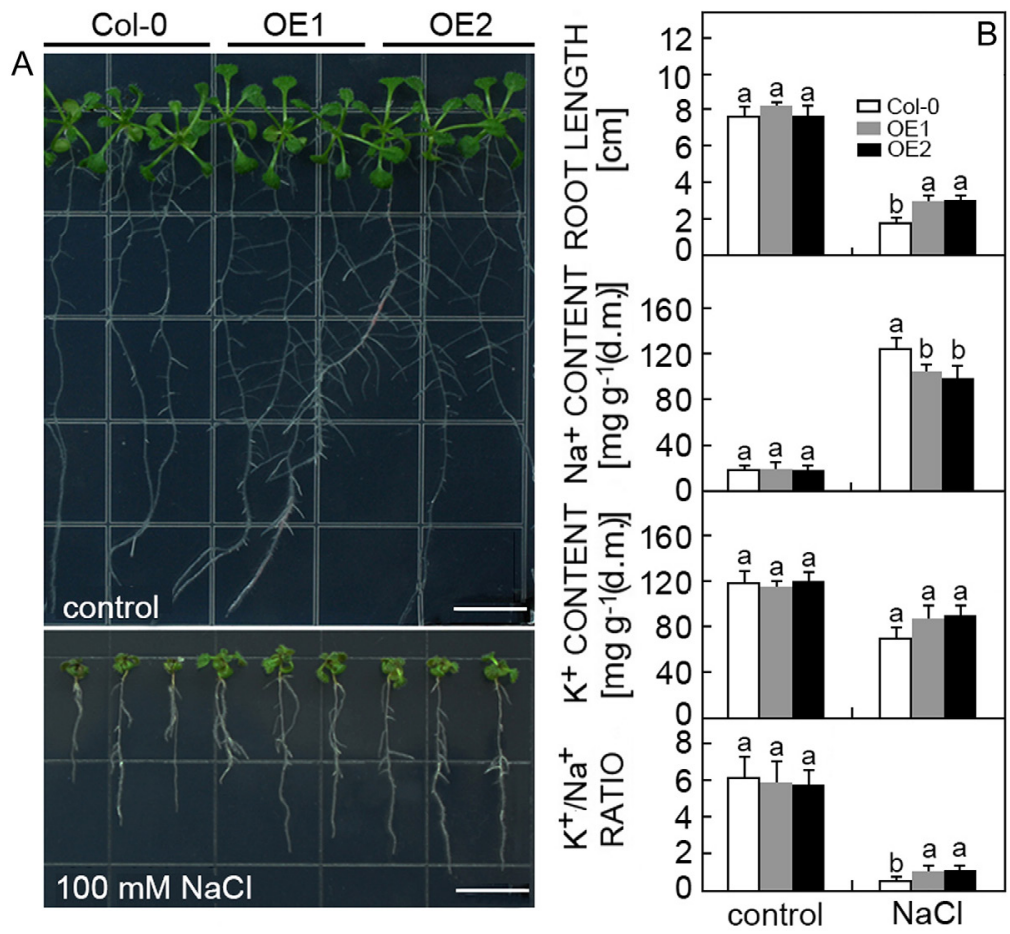

Fig. 3. Superoxide dismutase TaSOD5 ectopic overexpression offered salt tolerance in OE1 and OE2 transgenic lines in comparison with Col-0. A - Arabidopsis seedlings growing in plates with none and $100 \mathrm{mM} \mathrm{NaCl}$ (the bar is $1 \mathrm{~cm}$ ). $B$ - Root length, $\mathrm{Na}^{+}$content, $\mathrm{K}^{+}$content, $\mathrm{K}^{+} / \mathrm{Na}^{+}$ratio as affected by $\mathrm{NaCl}$. Means $\pm \mathrm{SDs}, n=3$, different letters indicate significant differences using one-way $A N O V A$ analysis $(P<0.05)$ 
many physiological processes, so the elevation of activities of ROS scavengers by TaSOD5 ectopic overexpression may affect ROS production system. Therefore, we also measured the activity of NOX, the major contributor to ROS production. When compared with Col- 0 , the OE lines had higher NOX activities (Fig. 4C).

\section{Discussion}

Over-amount of ROS leads to serious oxidative damage. To cope with this, plants have evolved the efficient and complicated ROS scavenging machinery. Within the machinery, SOD appears to play the central role because it converts highly toxic $\mathrm{O}_{2}^{-}$to low toxic $\mathrm{H}_{2} \mathrm{O}_{2}$ (Fridovich 1995). The ectopic overexpression of TaSOD5 enhanced the activities of total SOD and Cu/Zn SOD in Arabidopsis (Fig. 2C) indicating that TaSOD5 expression is connected with a $\mathrm{Cu} / \mathrm{Zn}$ SOD enzymatic activity.

Salt and other abiotic stresses often induce the production of ROS, so salt tolerance is partially attributed to the capacity of coping with ROS accumulation. Here, TaSOD5 ectopic overexpression enhanced tolerance to salinity and oxidative stress (Figs. 3, 4). In line with previous studies finding the increase in SOD activity by overexpressing $S O D$ genes enhances the tolerance to abiotic stress via reducing ROS accumulation (Allen 1995, Gill et al. 2015), our work demonstrates that TaSOD5 enhanced salt tolerance by increasing ROS removal ability.

Note that SOD catalyses the production of $\mathrm{H}_{2} \mathrm{O}_{2}$, so the increased tolerance to $\mathrm{H}_{2} \mathrm{O}_{2}$ suggests that downstream ROS scavengers were involved in the enhanced tolerance to salt stress in the TaSOD5 ectopic overexpression lines. Consistently, TaSOD5 ectopic overexpression elevated the activities of three $\mathrm{H}_{2} \mathrm{O}_{2}$ removal enzymes (Fig. 4C) indicating that SOD performs roles by comprehensively altering the ROS scavenging system. One possible cause is that the enhanced activities of these $\mathrm{H}_{2} \mathrm{O}_{2}$ removal enzymes reduce $\mathrm{H}_{2} \mathrm{O}_{2}$ content and therefore avoid the inhibition of SOD activity because $\mathrm{H}_{2} \mathrm{O}_{2}$ is an inhibitor of $\mathrm{SOD}$ (Allen 1995). The other one is that although $\mathrm{H}_{2} \mathrm{O}_{2}$ and $\mathrm{O}_{2}^{-}$are two moderately reactive ROS, they and can form highly reactive hydroxyl radicals through the HaberWeiss reaction (Apel and Hirt 2004). The opening question is how TaSOD performs the modulation.

Besides as toxic molecules, ROS also serve as signals to
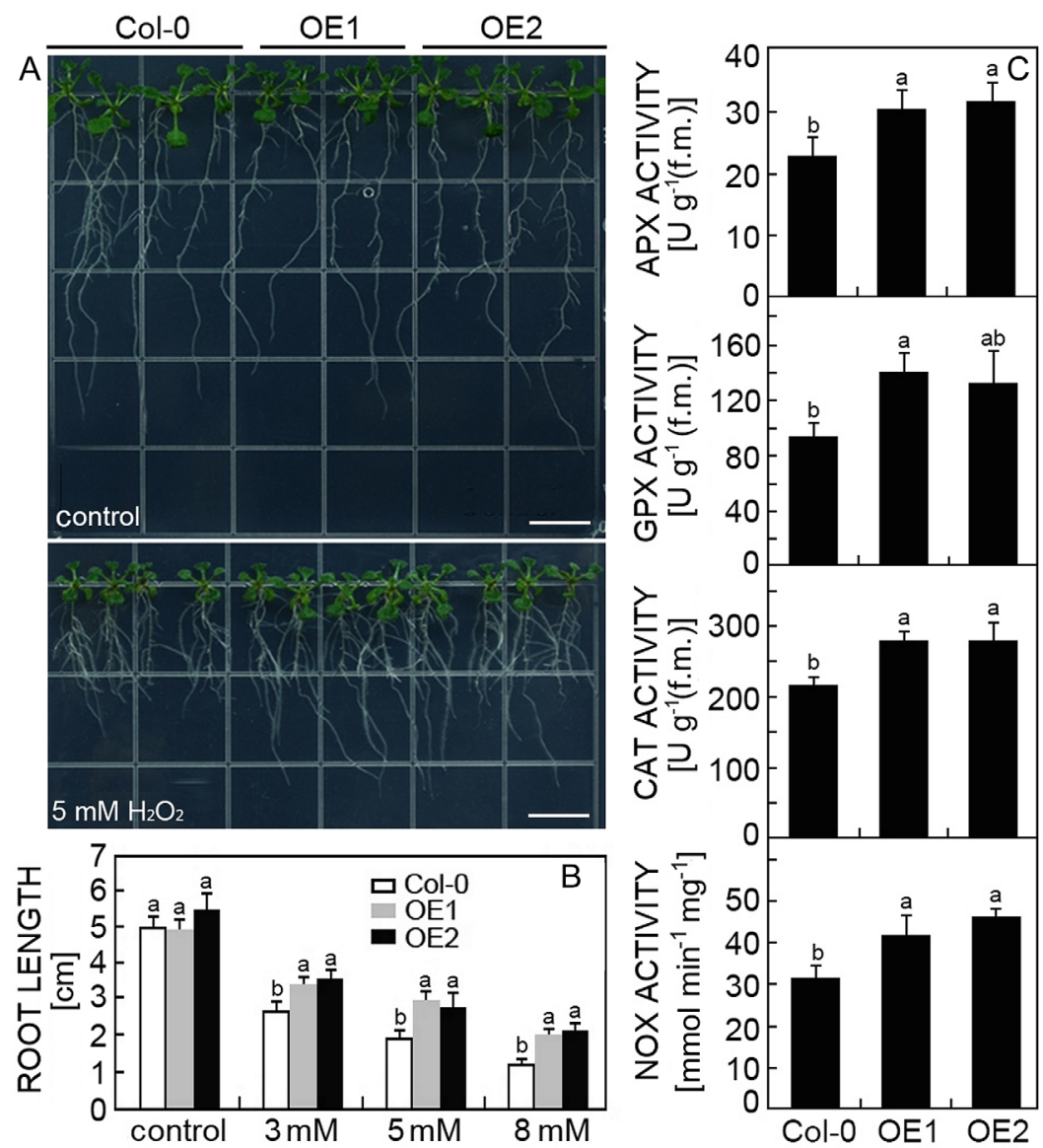

Fig. 4. Superoxide dismutase TaSOD5 ectopic overexpression enhanced tolerance to $\mathrm{H}_{2} \mathrm{O}_{2}$ and affected the activities of ROS scavengers ascorbate peroxidase (APX), glutathione peroxidase (GPX), and catalase (CAT) and also reactive oxygen species (ROS) producer NADPH oxidase (NOX). A - Arabidopsis seedlings growing in plates with none and $5 \mathrm{mM} \mathrm{H}_{2} \mathrm{O}_{2}$. B - Root length at different $\mathrm{H}_{2} \mathrm{O}_{2}$ concentrations. $C$-APX, GPX, CAT, and NOX activities. U - one unit of enzyme activity catalyzes the conversion of $1 \mu$ mol of substrate to product in $1 \mathrm{~min}$ at $25^{\circ} \mathrm{C}$ and $\mathrm{pH} 7.0$; Col-0 - Arabidopsis ecotype Col-0; OE1, OE2 - transgenic Arabidopsis lines over-expressing TaSOD5; the bar is $1 \mathrm{~cm})$. Means $\pm \mathrm{SDs}, n=3$, different letters indicate significant difference using one-way ANOVA $(P<0.05)$. 
regulate physiological processes, so suitable ROS amount is necessary for development of plants (Mittler et al. 2011, Suzuki et al. 2012, Sierla et al. 2013,). Control of ROS accumulation is orchestrated by the homeostasis between production and scavenging (Miller et al. 2009). The NADPH oxidase is the major ROS production enzyme that produces $\mathrm{O}_{2}{ }^{-}$(Torres and Dangl 2005, Suzuki et al. 2011), which is dismutated to $\mathrm{H}_{2} \mathrm{O}_{2}$ spontaneously or catalytically (Lin et al. 2009, Wi et al. 2012). Here, TaSOD5 ectopic overexpression increased the activity of NOX (Fig. 4C). In consistence with our finding, alteration of ROS scavengers has proved to affect the activity of ROS producers (Suzuki et al. 2013). These results indicate that SOD modulates not only the activities of the ROS scavenging system but also the ROS producer, confirming a close association between these two systems (Miller et al. 2009). Therefore, TaSOD5 ectopic overexpression enhanced salt and oxidative tolerance through ROS homeostasis modulation, which was achieved via promoting both the ROS scavenging system and the production system.

On the other hand, salt tolerance is comprehensively accomplished by multiple physiological processes including $\mathrm{Na}^{+}$exclusion towards the extracellular space or sequestration into the vacuole, better $\mathrm{K}^{+}$retention in root and leaf tissues, effective osmotic adjustment, and redox homeostasis (Munns and Tester 2008). Exactly, we found TaSOD5 ectopic overexpression lowered $\mathrm{Na}^{+}$ content but improved $\mathrm{K}^{+}$content and $\mathrm{K}^{+} / \mathrm{Na}^{+}$ratio in Arabidopsis under $\mathrm{NaCl}$ treatment. This might come from an increased antioxidation ability by which TaSOD5 ectopic overexpression attenuated the damage by oxidative stress, and therefore maintained a superior physiological status including ionic homeostasis maintenance capacity. Moreover, NOX has proved to modulate the mRNA stability of the Salt overly sensitive 1 , the crucial component of the Salt overly sensitive $1 / 2$ pathway (Chung et al. 2008); NOX-catalyzed ROS serve as signal molecules to regulate $\mathrm{Na}^{+} / \mathrm{K}^{+}$balance, and NOX mutants rbohd and rbohf are salt-sensitive and have high $\mathrm{Na}^{+} / \mathrm{K}^{+}$ratios under salt stress (Ma et al. 2011). The accumulated ROS is required for activating downstream signaling transduction pathways (Miller 2009). Thus, a lower $\mathrm{Na}^{+} / \mathrm{K}^{+}$ratio and a higher NOX activity by TaSOD5 ectopic overexpression suggest that TaSOD5 enhanced salt tolerance via mechanisms beyond modulating redox homeostasis alone.

It has been found that apart from positive contribution, SOD has different effects on tolerance to abiotic stresses. For example, in tobacco, the overexpression of a $S O D$ gene has no effect on tolerance to oxidative stress (Pitcher et al. 1991), but the ectopic overexpression of a pea SOD gene increases oxidative stress-induced membrane damage (Gupta et al. 1993). These findings suggest the complicated mechanisms governing the performance of SOD in response to abiotic stresses. This brings about a view that the causal link between antioxidant enzymes and overall tolerance to salt and other abiotic stresses is questioned and seriously criticized (Allen et al. 1995) because it was argued that salt-tolerance species do not need a higher antioxidation activity as they prevent formation of ROS in the first instance (Bose et al. 2014). On the other hand, a rapid burst of NOX-mediated ROS production is required for regulating downstream pathways and acclimation of plants to stress stimuli (Baxter et al. 2013). Thus, it could not be excluded that the improved SOD activity may reduce ROS amount to a level lower than a suitable threshold and therefore not enhance tolerance to oxidative stress.

In conclusion, TaSOD5 may play an important role in responding to salinity and oxidative stresses by enhancing the activities of total SOD and $\mathrm{Cu} / \mathrm{Zn}$ SOD, keeping a lower $\mathrm{Na}^{+} / \mathrm{K}^{+}$ratio and a high NOX activity, as well as promoting both ROS scavenging and the production system. However, the detailed regulatory mechanisms and signaling pathways are still unclear, and further research is needed in the future.

\section{References}

Allen, R.D.: Dissection of oxidative stress tolerance using transgenic plants. - Plant Physiol. 107: 1049-1054,1995.

Apel, K., Hirt, H.: Reactive oxygen species: metabolism, oxidative stress, and signal transduction. - Annu. Rev. Plant Biol. 55: 373-399, 2004.

Baxter, A., Mittler, R., Suzuki, N.: ROS as key players in plant stress signalling. - J. exp. Bot. 65:1229-1240, 2013.

Bose, J., Rodrigo-Moreno, A., Shabala, S.: ROS homeostasis in halophytes in the context of salinity stress tolerance. - J. exp. Bot. 65: 1241-1257, 2014.

Bradford, M,M.: A rapid and sensitive method for the quantitation of microgram quantities of protein utilizing the principle of protein-dye binding. - Anal. Biochem. 72: 248-254, 1976.

Chung, J,S., Zhu, J.K., Bressan, R.A., Hasegawa, P.M., Shi, H.Z.: Reactive oxygen species mediate Na-induced SOS1 mRNA stability in Arabidopsis. - Plant J. 53: 554-565, 2008.

Clough, S.J., Bent, A.F.: Floral dip: a simplified method for Agrobacterium-mediated transformation of Arabidopsis thaliana. - Plant J. 16: 735-743, 1998.

Dong, W., Ai, X., Xu, F., Quan, T., Liu, S., Xia, G.: Isolation and characterization of a bread wheat salinity responsive ERF transcription factor. - Gene 511: 38-45, 2012.

Dong, W., Liu, X., Li, D., Gao, T., Song, Y.: Transcriptional profiling reveals that a MYB transcription factor MsMYB4 contributes to the salinity stress response of alfalfa. - PLoS ONE 13: e0204033, 2018.

Dong, W., Wang, M., Xu, F., Quan, T., Peng, K., Xiao, L.: Wheat oxophytodienoate reductase gene TaOPRl confers salinity tolerance via enhancement of abscisic acid signaling and reactive oxygen species scavenging. - Plant Physiol. 161: 1217-1228, 2013.

Francois, L.E., Grieve, C.M., Maas, E.V., Lesch, S.M.: Time of salt stress affects growth and yield components of irrigated wheat. - Agron. J. 86: 100-107, 1994.

Fridovich, I.: Superoxide radical and superoxide dismutases. Annu. Rev. Biochem. 64: 97-112, 1995.

Ge, P., Hao, P., Cao, M., Guo, G., Lv, D., Subburaj, S., Li, X., Yan, X., Xiao, J., Ma, W., Yan, Y.: iTRAQ-based quantitative proteomic analysis reveals new metabolic pathways of wheat seedling growth under hydrogen peroxide stress. - Proteomics 13: 3046-3058, 2013.

Gill, S.S., Anjum, N.A., Gill, R., Yadav, S., Hasanuzzaman, M., Fujita, M., Mishra, P., Sabat, S.C., Tuteja, N.: Superoxide dismutase - mentor of abiotic stress tolerance in crop plants. Environ. Sci. Pollut. Res. Int. 22: 10375-10394, 2015.

Grace, S.C., Logan, B.A.: Acclimation of Foliar antioxidant 
systems to growth irradiance in three broad-leaved evergreen species. - Plant Physiol. 112: 1631-1640, 1996.

Gupta, A.S., Webb, R.P., Holaday, A.S., Allen, R.D.: Overexpression of superoxide dismutase protects plants from oxidative stress: induction of ascorbate peroxidase in superoxide dismutase-overexpressing plants. - Plant Physiol. 103: 1067-1073, 1993.

Hoagland, D.R., Arnon, D.I.: The water-culture method for growing plants without soil.- Univ. Calif. Exp. Sta. Circ. 347: $1-32,1950$.

Huang, Q., Wang, Y., Li, B., Chang, J., Chen, M., Li, K.: TaNAC29, a NAC transcription factor from wheat, enhances salt and drought tolerance in transgenic Arabidopsis. - BMC Plant Biol. 15: 268, 2015.

Huang, X., Madan, A.: CAP3: A DNA sequence assembly program. - Genome Res. 9: 868-877, 1999.

Ihsan, M.Z., El-Nakhlawy, F.S., Ismail, S.M., Fahad, S., Daur, I.: Wheat phenological development and growth studies as affected by drought and late season high temperature stress under arid environment. - Front Plant Sci. 7: 795, 2016.

Kumar, A., Logan, S., Matija, S., Neil, M.N., Hickerson, M.J., Marcus, A.S.: Abiotic stress signaling in wheat -an inclusive overview of hormonal interactions during abiotic stress responses in wheat. - Front Plant Sci. 9: 734, 2018.

Livak, K.J., Schmittgen, T.D.: Analysis of relative gene expression data using real-time quantitative PCR and the $2^{-\Delta \Delta C T}$ method. - Methods 25: 402-408, 2001.

Lin, F., Ding, H., Wang, J., Zhang, H., Zhang, A., Zhang, Y., Tan, M., Dong, W., Jiang, M.: Positive feedback regulation of maize NADPH oxidase by mitogen-activated protein kinase cascade in abscisic acid signalling. - J. exp. Bot. 60: 32213238, 2009.

Liu, S., Liu, S., Wang, M., Wei, T., Meng, C., Wang, M., Xia, G.: A wheat similar to $R C D-O N E$ gene enhances seedling growth and abiotic stress resistance by modulating redox homeostasis and maintaining genomic integrity. - Plant Cell 26: 164-180, 2014.

Ma, L., Zhang, H., Sun, L., Jiao, Y., Zhang, G., Miao, C., Hao, F.: NADPH oxidase AtrbohD and AtrbohF function in ROS dependent regulation of $\mathrm{Na}^{+} / \mathrm{K}^{+}$homeostasis in Arabidopsis under salt stress. - J. exp Bot. 63: 305-317, 2011.

Miller, G., Schlauch, K., Tam, R., Cortes, D., Torres, M.A., Shulaev, V., Dangl, J.L., Mittler, R.: The plant NADPH oxidase RBOHD mediates rapid systemic signaling in response to diverse stimuli. - Sci. Signal. 2: ra45, 2009.

Mittler, R.: Oxidative stress, antioxidants and stress tolerance. -
Trends Plant Sci. 7: 405-410, 2002.

Mittler, R., Vanderauwera, S., Suzuki, N., Miller, G., Tognetti, V.B., Vandepoele, K., Gollery, M., Shulaev, V., Van Breusegem, F.: ROS signaling: the new wave? - Trends Plant Sci. 16: 300-309, 2011.

Munns, R., Tester, M.: Mechanisms of salinity tolerance. - Annu. Rev. Plant Biol. 59: 651-681, 2008.

Murashige, T., Skoog, F.: A revised medium for rapid growth and bioassays with tobacco tissue cultures. - Physiol. Plant 15: 473-497,1962.

Pitcher, L.H., Brennan, E., Hurley, A., Dunsmuir, P., Tepperman, J.M., Zilinskas, B.A.: Overproduction of petunia copper/ zinc superoxide dismutase does not confer ozone tolerance in transgenic tobacco. - Plant Physiol. 97: 452-455, 1991.

Rong, W., Qi, L., Wang, A., Ye, X., Du, L., Liang, H., Xin, Z., Zhang, Z.: The ERF transcription factor TaERF3 promotes tolerance to salt and drought stresses in wheat. - Plant Biotechnol. J. 12: 468-479, 2014.

Sierla, M., Rahikainen, M., Salojärvi, J., Kangasjärvi, J., Kangasjärvi, S.: Apoplastic and chloroplastic redox signaling networks in plant stress responses. - Antioxid. Redox Signal 18: 2220-2239, 2013

Suzuki, N., Koussevitzky, S., Mittler, R., Miller, G.: ROS and redox signalling in the response of plants to abiotic stress. Plant Cell Environ. 35: 259-270, 2012.

Suzuki, N., Miller, G., Morales, J., Shulaev, V., Torres, M.A., Mittler, R.: Respiratory burst oxidases: the engines of ROS signaling. - Curr. Opin. Plant Biol. 14: 691-699, 2011.

Suzuki, N., Miller, G., Salazar, C., Mondal, H.A., Shulaev, E., Cortes, D.F., Shuman, J.L., Luo, X., Shah, J., Schlauch, K., Shulaev, V., Mittler, R.: Temporal-spatial interaction between reactive oxygen species and abscisic acid regulates rapid systemic acclimation in plants. - Plant Cell 25: 3553-3569, 2013.

Torres, M.A., Dangl, J.L.: Functions of the respiratory burst oxidase in biotic interactions, abiotic stress and development. - Curr. Opin. Plant Biol. 8: 397-403, 2005.

Wi, S.J., Ji, N.R., Park, K.Y.: Synergistic biosynthesis of biphasic ethylene and reactive oxygen species in response to hemibiotrophic Phytophthora parasitica in tobacco plants. Plant Physiol. 159: 251-265, 2012.

Zahid, H.S., Hafiz, M.R., Tasneem, A., Ihsanullah, D., Muhammad, A., Nawaz, M.Q., Ahmad, I.A., Rana, R.M., Atif, S.H., Yang, G.C.: Redox and ionic homeostasis regulations against oxidative, salinity and drought stress in wheat (a systems biology approach). - Front. Genet. 8: 141, 2017. 